\title{
Dan Zahavi and John Searle on Consciousness and Non-Reductive Materialism
}

\author{
AGUSTINA LOMBARDI \\ Ian Ramsey Centre for Science and Religion \\ University of Oxford \\ agustina.lombardi@theology.ox.ac.uk
}

\begin{abstract}
In his 1994 paper, neuroscientist Benjamin Libet affirmed that he found a way to test the interaction between the mind and the brain. He believed that this procedure would also test the reality of a non-physical mind, emerging from neural activity. In 2000 John Searle objected to Libet's evident dualism, affirming that the mind is not a hypothesis to test but a datum to be explained. According to Searle, Libet's problem arose from accepting the Cartesian distinction of 'mind' and 'body', obsolete categories from an old philosophy. Searle's solution implied the rejection both of dualism and reductive materialism, suggesting a non-reductive materialism. This first-person ontology, as Searle calls it, aims at preserving, against reductive materialism, the essential features of consciousness, i.e. subjective, qualitative and unified. Searle argued, however, that the nature of consciousness remained biological. Dan Zahavi's thesis is that a phenomenological approach to the self and consciousness can offer a complementing alternative to the contemporary comprehension of the nature of consciousness. Engaged in an open dialogue with neuroscience and cognitive science in his dealings with consciousness, Zahavi holds, nonetheless, that this notion cannot be completely understood without reference to the notion of the self. Thus, this paper will analyse Zahavi's ideas about the self and consciousness in Subjectivity and Selfhood (2005) as to evaluate whether his phenomenological approach is able to overcome the materialist tones of Searle's philosophy of mind, opening the possibility of a non-reductive comprehension of human beings.
\end{abstract}

Keywords: consciousness; mind; dualism. 


\section{Introduction}

The past couple of decades have increasingly shown scientifically informed philosophical and theological discussions surrounding the nature of consciousness and its relation to the brain - the mind/body problem -, within the analytic and phenomenological traditions. Neuroscientist Benjamin Libet, for example, designed in 1994 an experiment to explain their relationship suggesting the existence of a non-physical conscious mental field (CMF) with causal powers over neural activity (Libet 1994). Libet invites us to consider the possibility of the following experiment to demonstrate the existence of the CMF:

If local areas of cerebral cortex could independently contribute to or alter the larger, unitary CMF, it should be possible to demonstrate such contributions when (a) that cortical area is completely isolated or cut off from neuronal communication with the rest of the brain, but (b) the area remains in situ, alive and kept functioning in some suitable manner that sufficiently resembles its normal behaviour. The experimental prediction to be tested would be as follows: Suitable electrical and/or chemical activation of the isolated tissue should produce or affect a conscious experience, even though the tissue has no neural connections to the rest of the brain. Possibilities of spread of influences from the isolated block via physical non-neural paths (e.g. electric current flow) would have to be controlled for. If a subjective experience is induced and reportable within a second or so, that would tend to exclude spread by chemical diffusion or by changes in vascular circulation or in contents of circulating blood as a cause. [...] A slab of cerebral cortex can be neurally isolated surgically, remaining in place but viable by retaining its blood supply as the only connection with the rest of the brain. This is accomplished by making all of the cuts subpially. Studies of the electrophysiological activity of such isolated cortex in situ have been reported. The basic method involved introducing a narrow curved blade through an opening in an avascular area of the pia-arachnoid membrane. This could undercut a block or slab of cortex and, by bringing its tip up to meet the pia at some distance away, also cut the connetions to adjacent cortex. [...] If stimulation of the isolated cortical slab can elicit an introspective report by the subject, that could only come about if the CMF could activate the appropriate cerebral areas required to produce the verbal report. (Libet 1994, 121-124) 
Clearly, it would take much to convince any candidate to undergo such excision, which renders the experiment almost unfeasible. Philosophically, it resulted in numerous accusations against Libet of supporting a naïve Cartesian dualism. The analytic philosophical tradition engaged most prominently with the scientific discourses on these issues, for example in the writings of John Searle, who discusses some discoveries in neuroscience to develop his own approach to consciousness. Searle affirms that the Cartesian notions of 'mind' and 'body', the explananda in Libet's experiments, are obsolete categories from an old philosophy, which need to be rejected in favour of a type of non-reductive materialism. Within the phenomenological tradition, Dan Zahavi and his colleague Shaun Gallagher openly engage with contemporary neuroscience and cognitive science as well. As in the case of Searle, they also reject Cartesian dualism, claiming that instead of explaining how the mind interacts with the body, phenomenology overcomes Cartesian dualism by changing the focus and presenting the notion of the embodied self.

Acknowledging that this is certainly a complex question, perhaps one of the most difficult problems in philosophy and theology, the goal of this paper is to analyse critically these two different anti-Cartesian approaches to the problem of consciousness and body, creating a dialogue between Searle and Zahavi, who represent the analytic and the phenomenological traditions respectively, in the hope of building a bridge between these approaches. I will focus on the notion of consciousness and self-consciousness. Following Zahavi, I hold that a phenomenological approach to consciousness can offer a complementing alternative to the contemporary analytic understanding of the nature of consciousness and self-consciousness. In addition to analysing these claims, I will evaluate whether his phenomenological approach opens the possibility of overcoming the current materialist cast of Searle's philosophy of mind, allowing for the possibility of a non-reductive comprehension of human beings.

The strategy followed is three-fold. First, I will frame the notion of consciousness in the mind/body problem as presented by Searle. This step will also include an analysis of Searle's understanding of the structure of consciousness, which arises from his willingness to overcome both Cartesian 
dualism and eliminative materialism (or reductive materialism, as he also calls it - Searle 2004,56 ) in dialogue with contemporary neuroscience. Afterwards, I will turn my attention to Zahavi's phenomenological approach. Even though he does not directly engage with the debate over the materiality or immateriality of consciousness, as Searle does, he engages with the debate concerning Cartesian dualism. Hence, I will present his views on consciousness as an embodied self-consciousness, which imply that it is not an object among other objects in the world. As I will explain, Zahavi embraces this notion of consciousness to give way to the possibility of a world full of meaningful objects. Finally, I will argue that, even if Zahavi does not engage in ontological questions about consciousness, his account leaves a path open to understanding it in non-material terms, while being intrinsically related to the body, overcoming, thus, an extrinsic Cartesian dualism.

\section{Searle's non-reductive materialism}

Throughout much of his work, John Searle approaches consciousness by engaging with scientific discoveries and developing his own naturalistic perspective. Against many scientists, including Libet, he holds that consciousness "is not a hypothesis that has to be tested but a datum that has to be explained" (Searle 2001, 63). Indeed, according to Searle, the question is not whether consciousness exists but "how brain processes cause a conscious field and how such experiences are realized in the brain" (Searle 2001, 63). Thus, there is no need for proving the existence of consciousness, since we experience it, but to explain the experience of its existence. Searle's problem with Libet's outlook is that he postulates a non-physical CMF. Even though Libet understands CMF to emerge from neural activity, he claims that it belongs to a subjective non-physical realm: "The putative CMF would not be in any category of known physical fields, such as electromagnetic, gravitational, etc. The conscious mental field would be in a phenomenologically independent category; it is not describable in terms of any externally observable physical events or of any known physical theory as presented constituted" (Libet 1994, 120). 
Searle's main concern is that Libet evidently embraces dualism: "[Libet] thinks that in recognizing the unified character of normal conscious experiences we are postulating the existence of something 'non-physical', we are postulating some weird Cartesian entity with separate causal powers, not a part of the ordinary, 'physical', biological world" (Searle 2001, 63). Instead, Searle proposes a new way of understanding the relation between consciousness and the brain that would, in some way, overcome this naïve dualism. His claim is that Libet, together with all philosophies of mind (all -isms, he says), bases his views on a wrong assumption, namely the distinction between the mental and the physical or material (Searle 2001, 1-3). Consciousness is, for Searle, a biological process as any other, which presents certain specific features that allow him to distance himself from reductive materialism, as I will show briefly.

Searle complains that philosophy of mind typically offers two options: one can either be a dualist or an eliminative materialist (in any of its alternatives: functionalism, behaviourism, etc). The problem is that both dualism and materialism accept the traditional vocabulary of 'mind' and 'body'. Searle simply rejects the use of this obsolete vocabulary, implying thus the rejection of both dualism and reductive materialism. On the one hand, he rejects dualism because he considers the postulation of a non-physical mental realm to be both unnecessary and contradictory to what we know about the way the universe functions. On the other, he rejects eliminative materialism, because it denies the existence of some essential features of the mind, such as consciousness and intentionality.

Instead, Searle suggests embracing a non-reductive materialism. He claims to provide a naturalistic solution to the mind/body problem, holding that mental states are biological processes going on in our brains, simultaneously accepting the existence of qualitativeness, subjectivity, unity, and intentionality, among other essential features of consciousness (Searle 2004, 93-110, where he describes eleven of these essential features of consciousness). Searle calls his solution a 'biological naturalism', the principal goal of which is to "abandon the assumptions behind the traditional vocabulary" (Searle 2004, 73). The main assumption that we have to overcome, Searle 
clarifies, is that which holds that 'mental' and 'physical' are categories that exclude each other (Searle 2004, 76). Once one realises that there is no deep metaphysical distinction between them, the apparent problem of the mind/ body interaction is solved. Moreover, once one understands the underlying biological process, the mystery is over:

It should seem no more mysterious, in principle, that this hunk of matter, this grey and white oatmeal-textured substance of the brain, should be conscious than it seems mysterious that this other hunk of matter, this collection of nucleo-protein molecules stuck onto a calcium frame, should be alive. The way, in short, to dispel the mystery is to understand the process. We do not yet fully understand the process, but we understand their general character. (Searle 1984, p. 24. See also Searle 1992, 100, and 2004, 80-82)

The mystery of the living body is for Searle similar to the mystery of the conscious brain, both of which will be solved when science fully discovers their respective underlying processes. As part of the physical world, consciousness is caused by lower-level physical processes, which in this case are neurological processes that occur in the brain: "Consciousness is a system-level, biological feature in much the same way that digestion, or growth, or the secretion of bile are system level, biological features. As such, consciousness is a feature of the brain and thus a part of the physical world" (Searle 2004, 80). The key for Searle is that even though consciousness is a physical reality, there is no contradiction in saying that it is subjective, qualitative, and intentional. Hence, these features are, as well, part of the domain of the natural sciences. Nonetheless, neither consciousness nor its features can be ontologically reduced to a non-subjective reality, even though they can be causally reduced, i.e. explained by their neuronal substrate. This claim does not intend to show a "deep metaphysical asymmetry" (Searle 2004, 85), but it wants to affirm that consciousness has a first-person ontology that cannot be reduced to a third-person perspective.

In summary, Searle's position amounts to affirming the irreducible subjectivity of consciousness side by side the physicality of all human 
existence. A similar account has also been suggested, with its own distinctive features, by phenomenology. Within this tradition, Zahavi is today one of its most prominent proponents, building dialogical bridges with neuroscience and the analytic tradition. I will now turn my attention to his ideas, as to elucidate his notion of consciousness and assess its merits in overcoming Cartesian dualism without reducing consciousness to biological processes.

\section{An alternative phenomenological approach}

Among phenomenologists familiar with Zahavi's work, many would wonder the reasons for introducing his phenomenological approach to the discussion of the mind/body problem and the nature of consciousness. After all, Zahavi is explicit when claiming that metaphysical discussions distort the object under study when phenomenology engages with the problem of consciousness. Indeed, he holds that the starting point of many philosophers and theologians consists in describing metaphysical positions. In fact, Zahavi and Gallagher affirm that "one of the underlying ideas of phenomenology is that the preoccupation with these metaphysical issues tends to degenerate into highly technical and abstract discussions that lose touch with the real subject matter: experience" (Gallagher \& Zahavi 2008 , 9). Phenomenology, instead, is mainly concerned with a description of the "experiential structure of our mental/embodied life" (Gallagher \& Zahavi 2008, 9), from a first-person perspective. Moreover, phenomenology, they claim, is not interested in giving a naturalistic explanation of consciousness, but neither is it interested in rejecting its importance: "Don't we have to know whether we are studying the mind, or the brain, or whether it is something material or immaterial? ... Phenomenologists do not deny it, nor do they affirm it. They suspend these kinds of questions and all judgements about them. They start with experience" (Gallagher \& Zahavi 2008, 7).

Moreover, as a good analytic philosopher, Searle also rejects using phenomenology to address ontological questions: "The phenomenological approach is inadequate to solve the problems that bother me. For me the 
phenomenological data are typically useful at the beginning of the investigation, logical analysis has to go far beyond phenomenology" (Searle 2001/2002, 277). Searle claims that phenomenology has severe limitations given that it only provides a description of how things seem to me here and now, while he is interested in analysing their ontology, i.e. what is the nature of consciousness.

So, again, why introducing Dan Zahavi's views? In brief, because I think that he can offer valuable insights, which Zahavi himself might not have seen, concerning the ontological questions about the nature of consciousness, while at the same time changing the focus of the mind/body problem away from Cartesian considerations. Starting from experience, i.e. the phenomenon as it appears to us, suspending any influential metaphysical or theoretical framework, and following fully the phenomenological method as it was taught by Husserl, in particular the third step of the method, namely the eidetic variation, I believe that Zahavi's understanding of consciousness might open a path for the consideration of a non-material realm in human beings. Thus, I will present his views on consciousness, in order to offer my views on this matter.

Zahavi begins his analysis of consciousness establishing the relationship between consciousness and self-consciousness. One must not identify self-consciousness with something that occurs only when one pays attention to conscious life. Rather, every conscious experience contains a structural minimal form of self-consciousness. This means that there is a "constitutive link between experiential phenomena and first-personal givenness or accessibility" (Zahavi 2005, 12), i.e., every conscious experience is my experience because it has an undeniable first-person perspective: "insofar as there is something it is like for the subject to have the experience, the subject must in some way have access to and be acquainted with the experience" (Zahavi 2005, 15). In fact, we are acquainted with our own subjectivity in a way completely different from how we are acquainted with objects. Thus, we can have various different experiences of different things, but all these experiences have in common that they have a 'mineness' quality, i.e. they are experienced as mine. Furthermore, I do not possess those experiences as 
I possess external objects: "the for-me-ness in question is not a quality like yellow, salty, or spongy. It doesn't refer to a specific content of experience, to a specific what, but to the unique mode of givenness or how of experience. It refers to the first-personal presence of experience; it refers to the fact that experiences I am living through present themselves differently (but not necessarily better) to me than to anybody else" (Gallagher \& Zahavi $2008,57)$. Experiences, thus, present themselves in a perspectival sense, in a distinctive manner to the experiencing subject.

Moreover, following Jean-Paul Sartre, Zahavi holds that this self-consciousness (or self-awareness, as many phenomenologists speak) must not be mistaken for an additional mental state such as reflection or introspection, but should be accepted as an intrinsic feature of primary experience. Whereas in reflection consciousness takes itself as the object, in the primary self-awareness (which Sartre calls pre-reflective self-awareness), consciousness has a non-objectifying self-awareness. Reflection, which is a derived self-awareness, always presupposes pre-reflective self-awareness. Thus, consciousness has no need of reflection for being acquainted with itself. Zahavi gives an example to illustrate this idea. Suppose, he says, that you are reading a book, and that someone interrupts you to ask what you are doing. Even though your attention has not been focused on yourself nor in the activity of reading but in the story itself, you can answer ' $I$ am reading'. The reason for that, he holds, is that during your activity, you have been conscious of yourself all along. For Searle, on the contrary, even if he postulates a first-person ontology in the sense that a subject always experiences mental phenomena, he rejects the phenomenological notion of self-consciousness. For Searle, to be self-conscious is to have a kind of reflection, is to turn the attention to the state itself. Therefore, not every conscious event is self-conscious (Searle 2004, 142-149). Nonetheless, Zahavi affirms that having a primitive and fundamental pre-reflective consciousness is having a 'what it is like' experience that refers to a subject, which, using Searle's terminology, is to claim a first-person ontology.

David Rosenthal objected the assertion that there is an intrinsic feature belonging to the primary experience (Rosenthal 1993, 157). Rosenthal argued 
that to postulate self-consciousness as an intrinsic feature is to postulate an 'unanalysable' and 'mysterious' feature that cannot be studied scientifically nor theoretically. Zahavi's answer to this objection will allow me to assess the possibility of a naturalistic interpretation of consciousness, which basically refers to Searle's own programme. When discussing the postulation of the intrinsic pre-reflective self-consciousness, Zahavi asks:

Is a one-level account of consciousness committed to some kind of supernatural dualism? Not at all. One can defend the notion of pre-reflective self-consciousness while remaining quite neutral vis-à-vis the issue of naturalization. More specifically, nothing in the rejection of a relational account of consciousness rules it out that the emergence of consciousness requires a requisite neural substratum... It might even be argued that the one-level account is... in better accordance with a popular view in neuroscience, according to which consciousness is a matter of hitting a certain threshold of neural activity. (Gallagher \& Zahavi 2008, 63)

The key word in this statement is 'neutral'. Following this neutrality, it might be claimed, on the one hand, that this view of consciousness is in accordance with a naturalisation of consciousness, but, on the other hand, it might also be claimed that it is not. I am inclined to favour this second option, and I am also inclined to say that Zahavi's ideas might allow me to conclude this way. Recurring to the idea that consciousness is not another object in the world, I will show why I believe this statement to be true.

Zahavi holds that "any assessment of the possibility of reducing consciousness to neuronal structures and any appraisal of whether a naturalization of consciousness is possible will require a detailed analysis and description of the experiential aspects of consciousness" (Zahavi 2005, 4). This means that, if we want to reduce consciousness to neuronal processes, we first need to understand what we are reducing. According to Zahavi, phenomenologists claim that consciousness is not an object among others in the world, given that consciousness has an active role in the giving of meaning to things that appear to it. Consciousness is, Zahavi explains, "a necessary (though not sufficient) condition of possibility for any entity 
to appear as an object in the way it does and with the meaning it has" (Gallagher \& Zahavi 2008, 27). In this way, consciousness is distinguished from the objects of the world. Because of this specific characteristic of consciousness, Zahavi argues that phenomenologists do not attempt to find a place for consciousness in nature:

The phenomenological investigation of consciousness is not motivated by the wish to find a place for consciousness within an already well-established materialistic or naturalistic framework. In fact, the very attempt to do so, assuming that consciousness is merely yet another object in the world, would prevent one from discovering and clarifying some of the most interesting aspects of consciousness, including the true epistemic and ontological significance of the first-person perspective. The question of consciousness should not be addressed on the background of an unquestioned objectivism. Too frequently the assumption has been that a better understanding of the physical world will allow us a better understanding of consciousness. (Gallagher \& Zahavi 2008, 28)

Therefore, if consciousness is not like any other object in the world, a serious analysis of consciousness cannot be restricted to a neurobiological approach, as Searle pretends to do: "the neurobiological attempts to solve the scientific problem of consciousness... this is exactly the right approach" (Searle 2004, 105). Thus, even though Searle wants to retain many features of consciousness, including subjectivity, I believe that he is mistaken when he thinks that a complete science of the brain will resolve the mind/body problem and will give us a complete account of what consciousness is. Consciousness, as Zahavi and other phenomenologists argue, is not a thing that can be classified among the physical objects of the world. Consciousness is our access to the world, is what gives meaning to the objects that manifest and present themselves: "consciousness constitutes the way in which the world appears as meaningful” (Gallagher \& Zahavi 2008, 26). If we reduce the study of consciousness to a physical/biological explanation, how can we explain that neurons give meaning to things? Ultimately, how can a material res give meaning to objects? 


\section{Towards a non-materialistic overcoming of Cartesian dualism}

If consciousness is not a thing among objects in the world, can we speak in any meaningful way of an immaterial consciousness? The key issue in answering this question is whether an affirmative reply implies Cartesian dualism. I believe it does not. The main problem with Cartesian dualism is that it understands the mind/body relation in terms of an extrinsic relation between two distinct substances. This is the problem Libet tries to solve when he explains how the non-physical mind relates and interacts with the brain. Searle reacts to it eliminating the Cartesian res cogitans, while retaining the term 'mind'. Keeping the Cartesian res extensa (i.e. the material or physical thing that falls under the field of study of the natural sciences, such as physics, chemistry, or biology which, following Searle's grounding of this notion in contemporary physical conceptions, can be understood as essentially belonging to space and time, and explained by microphysics), Searle expands its features by characterising it as a subjective, qualitative, and intentional matter. I want to argue that, even if Searle changes the very notion of res extensa, his thought still remains constrained within the framework of the dualist/materialist long-standing debate. Indeed, he understands the relation between a non-material mind and the body as an extrinsic and imposed relation, forcing him to think in Cartesian terms, even if his notion of body differs from that of Descartes'. Ultimately, this framework prevents Searle from fulfilling his goal of overcoming the debate as a whole.

What I propose, following Zahavi's phenomenological approach, is to change the focus of the mind/body problem. The phenomenological method replaces the notions of 'mind' and 'body' ("obsolete vocabulary", according to Searle, "derivations and abstractions", according to Zahavi, given that the subject does not identify neither with her consciousness nor with her body in isolation) with the notion of 'embodiment', thus presenting a completely new paradigm in the discussion. Whereas most analytic philosophers, including Searle, begin the mind/body discussion presenting the different metaphysical alternatives and taking sides, Zahavi holds that "phenomenology pushes 
these kinds of questions aside, brackets them, sets them out of play, and asks us instead to pay attention to the phenomenon under study" (Gallagher \& Zahavi 2008, 6). As I explained above, the starting point of phenomenology is experience, which ultimately is an experience that unquestionably refers to the body, an embodied experience.

As with consciousness, the own body is not experienced as an object among other objects in the world. Following the teachings of Husserl, who distinguished between Körper (the objective body) and Leib (the lived body), paradigmatically appropriated by Merleau-Ponty's distinction between le corps objectif and le corps vécu, phenomenologists argue that there are two ways in which we can experience our own body. First, we can experience it from a third-person perspective, as from the outside. Second, from an embodied first-person perspective. While the former perceives the body as an object, subject for example to the study of the natural sciences, the latter is focused on the experienced body, as it appears to phenomenological investigation.

Zahavi explains that the lived body is perceived as "the body as subject, as experiencer, as agent, rather than the body as object, as thing experienced - this is a basic distinction missed by the Cartesian tradition" (Gallagher \& Zahavi 2008, 155). Thus, whereas Descartes believes that he can think without his body, because he is not his body, for phenomenology the body itself structures experience. We are embodied beings, and our body shapes our being-in-the-world. In addition to being involved in our relation to the world, our body is also involved in our relation to others. Indeed, our encounter with the other, the possibility of our mutual interaction, is only possible because of our embodied subjectivity. In fact, the other is perceived not as a mere body, but as another embodied subject, precisely because of my own embodied conscious experience. As Zahavi explains "we should avoid construing the mind as something visible to only one person and invisible to everyone else. The mind is not something exclusively inner, something cut off from the body and the surrounding world" (Zahavi 2005, 152). Thus, we perceive the other as a unified whole, i.e. as a conscious body.

With this kind of argumentation phenomenology opposes and rejects Cartesian dualism. Nonetheless, Zahavi argues that "this does not entail 
an endorsement of some kind of Cartesian materialism" either. In fact, he continues, "it is not as if the phenomenological way to 'overcome' dualism is by retaining the distinction between mind and body, and then simply getting rid of the mind. Rather, the notion of embodiment, the notion of an embodied mind or a minded body, is meant to replace the ordinary notions of mind and body" (Gallagher \& Zahavi 2008, 153). Therefore, phenomenology rejects the very distinction of res cogitans and res extensa, where the body is seen as a mere object and not as a constituent feature of our conscious self.

Zahavi does not go further than that. In fact, he avoids any mention of an immaterial realm in human beings. Probably due to his interests in engaging in dialogue with analytic philosophy of mind, in particular with Searle, Zahavi misses the opportunity to follow through the path he prepared with his phenomenological account of the conscious body. This shortcoming allows Searle to formulate his critique of phenomenology, namely that phenomenologists are not interested in ontological questions. In Zahavi's case, I believe this critique to be accurate. Nevertheless, when Husserl introduced the eidetic variation in his phenomenological method, he presented it as "a way that would draw out the essential and invariant characteristics of the things that we experience" (Gallagher \& Zahavi 2008, 30). It is in this final step where I think that phenomenology, starting with experience, opens itself to ontological questions.

Following Husserl's method, phenomenology must formulate the question about these essential and invariant features of consciousness, what I consider to be the core set of properties that remain despite change. There must be some essential features of human consciousness, at which one can arrive subtracting the variable characteristics. Within those core features, consciousness must be either material or immaterial. This is the ultimate question that Zahavi fails to address, as I said, probably due to his reluctance to be rejected by the analytic philosophers. If, then, phenomenology is a valuable method to overcome Cartesian dualism without getting rid of an immaterial mind, how should we conceive of this immaterial consciousness? Indeed, phenomenology rejects Cartesian dualism understood in terms of an extrinsic relation between two completely distinct res, as in the case of Libet. 
But, would it make sense to affirm the existence of both realities internally and intrinsically related to each other, without the need of reducing the mind to biological processes, as Searle does? I believe that phenomenology allows us to consider this possibility. The key notions are the 'lived body', i.e. the 'own body', and the 'meaning-giving' capacity of consciousness. The former would become where man's material and spiritual realms are intrinsically unified, implying their embodied unity. This lived body, which is a conscious body, is our access to the world and to the other, who is also perceived as an embodied subject, another conscious body. This perception of the other can only be achieved if the other is also perceived as meaningful, in the sense that it appears to be an intentional self capable also of providing things with meaning, precisely because she is also a conscious body. It is in examining this feature of our own consciousness, which first understands others as conscious and meaningful, where we can see that our consciousness also gives meaning to objects, which are not 'others', in the sense that objects, as well as 'others', appear to us in a certain way, according to our own first-person perspective. Thus, if we as embodied consciousness can give meaning to objects, it seems that this embodied consciousness cannot be of the same fabric of non-conscious objects. Hence, the meaning-giving capacity of these embodied subjects appears to refer to some non-material (non-objective) realm, in which our consciousness exists.

\section{Conclusion}

Starting from Searle's critique of Libet's Cartesian dualism and his account of non-reductive materialism, I suggested that his discussion remains constrained within a Cartesian framework, rendering his efforts unsuccessful, due to the fact that he thinks the relation between mind and body in any dualistic account as an extrinsic kind of relation. Thus he reduces the res cogitans to a res extensa with extra features, naturalising consciousness in terms of biological and neuronal processes. I proposed studying Zahavi's phenomenological take on consciousness as a way to re-consider Searle's position and to overcome Cartesian dualism. Zahavi's account offers a view 
of consciousness that features an emphasis on the lived body and on it not being another object in the world. Even if Zahavi does not allow himself to continue his analysis of these ideas, hence not dealing with the question on the materiality or immateriality of consciousness, I argued that this is a valid and fruitful path to follow. Indeed, I suggested that these features permit to think of consciousness as something that is essentially irreducible to objective matter, even if it is intrinsically related to it, offering the possibility of interpreting it as belonging to a non-material realm, without rendering this position an extrinsic Cartesian dualism.

\section{References}

Gallagher, S., \& Zahavi, D. 2008. The Phenomenological Mind. An Introduction to Philosophy of Mind and Cognitive Science. New York: Routledge.

Libet, B. 1994. “A Testable Field Theory of Mind-Brain Interaction.” Journal of Consciousness Studies 1(1):119-126.

Libet, B. 2001. “'Consciousness, Free Action and the Brain'. Commentary on John Searle's Article (with a short reply from John R. Searle).” Journal of Consciousness Studies 8, 8:59-65.

Rosenthal, D. 1993. "Higher-order Thoughts and the Appendage Theory of Consciousness." Philosophical Psychology 6:155-166.

Searle, J. 1984. Mind, Brains and Science. Cambridge-Ma: Harvard University Press. Searle, J. 1992. The Rediscovery of the Mind. Cambridge-Ma: The MIT Press.

Searle, J. 2000. “Consciousness, Free Action and the Brain.” Journal of Consciousness Studies 7, 10:3-22.

Searle, J. 2001. “'Further Reply to Libet'. In Libet, B. 'Consciousness, Free Action and the Brain'. Commentary on John Searle's Article." Journal of Consciousness Studies 8, 8:63-65.

Searle, J. 2001/2002. "Neither Phenomenological Description Nor Rational Desconstruction: Reply to Dreyfus.” Revue internationale de philosophie 216:277-297.

Searle, J. 2004. Mind: A Brief Introduction. New York: Oxford University Press.

Zahavi, D. 2002. "First-person thoughts and embodied self-awareness: Some reflections on the relation between recent analytical philosophy and phenomenology." Phenomenology and the Cognitive Sciences 1:7-26.

Zahavi, D. 2004. "Introduction: Subjectivity in the Center or Back to Basics.” Phenomenology and the Cognitive Sciences 3:229-234.

Zahavi, D. 2005. Subjectivity and Selfhood. Investigating the First-Person Perspective. Massachusetts: The MIT Press. 